\title{
Kaposi's sarcoma concealed by stasis dermatitis in a patient with psoriasis
}

\author{
Hilal Kaya Erdoğan ${ }^{1 凶}$, Işıl Bulur², Zeynep Nurhan Saraçoğlu¹, Tekden Karapınar³, Deniz Arık ${ }^{4}$
}

\begin{abstract}
Kaposi's sarcoma (KS) is a multifocal and angioproliferative neoplasm. KS may be accompanied by psoriasis; however, in most of these cases the main mechanism involves iatrogenic KS associated with the immunosuppressive drugs that are used in psoriasis treatment. In angioproliferative lesions as a result of venous insufficiency and stasis dermatitis, acroangiodermatitis (pseudo-KS) is initially considered. However, the concurrent occurrence of psoriasis, stasis dermatitis, and KS has not been previously reported. We report a case of classic-type KS in an 83-year-old man that was concealed by stasis dermatitis and accompanied by psoriasis.
\end{abstract}

Keywords: psoriasis, Kaposi's sarcoma, stasis dermatitis

Received: 29 May 2017 | Returned for modification: 4 June 2017 | Accepted: 6 June 2017

\section{Introduction}

Kaposi's sarcoma (KS) is a multifocal angioproliferative neoplasm caused by infection with human herpesvirus 8 (HHV-8), also known as Kaposi's sarcoma (KS)-associated herpesvirus. Four different types of the disease have been described thus far, including classic (Mediterranean), endemic (African), iatrogenic (associated with immunosuppression), and epidemic KS (associated with human immunodeficiency virus, HIV) (1).

In some published cases, KS is accompanied by psoriasis; however, the main mechanism in most cases involves iatrogenic KS associated with the immunosuppressive drugs used in psoriasis treatment (1-5).

In angioproliferative lesions due to venous insufficiency and stasis dermatitis, acroangiodermatitis (pseudo-KS) is initially considered. However, the concurrent occurrence of psoriasis, stasis dermatitis, and KS has not been reported. Here, we describe a patient with classic-type KS concealed by stasis dermatitis and accompanied by psoriasis.

\section{Case report}

An 83-year-old male patient presented to our outpatient department for exudative wounds on his legs. He had a 10-year history of psoriasis and a 2-year history of reddish-purple papules on both legs. He had received hyperbaric oxygen therapy for 1 year for a venous ulcer, which had disappeared after the treatment. His medical history was unremarkable except for psoriasis and venous insufficiency. He was treated with topical steroids and calcipotriol, but did not undergo phototherapy or systemic treatment. He had no history of surgery or drug use, or any family history of any specific condition.

On dermatological examination, we noted erythematous, squamous plaques on both knees and elbows, and livid purple papules and nodules on both legs. Erythematous exudative lesions covered by yellow crusts consistent with stasis dermatitis were also observed (Fig. 1). Subungual hyperkeratosis and yellow discoloration were observed on his toenails. There were no pathological findings on laboratory examinations and the patient tested HIV-negative.
Two punch biopsies were performed based on the prediagnosis of psoriasis from the psoriasis plaque on his knee, and the prediagnosis of pseudo-KS and KS from the purple papules. Examination of the biopsy specimen obtained from the knee revealed regular acanthosis and hypogranulosis, mild spongiosis, and intraepidermal clusters of neutrophils (Munro's microabscess) in the epidermis, and the diagnosis of psoriasis was confirmed. Histopathological examination of the biopsy specimen obtained from the leg showed a lesion containing multiple vascular structures with various diameters (some of which were fissured) in the dermis, as well as mild nuclear hypertrophy and pleomorphism in the cells (Fig. 2). Immunohistochemical assays indicated a positive result for HHV-8 (Fig. 3). Thus, the patient was diagnosed with classic KS on the basis of the clinical, histopathological, and immunohistochemical findings.

The patient was referred to the oncology and radiation oncology departments for consultation. No findings suggesting internal organ or lymph node involvement were detected with positron emission tomography. Regression was observed in the lesions with radiotherapy. He continues to be followed and treated at our clinic and the radiation oncology department.



Figure 1 | Erythematous, squamous, psoriatic plaques, livid purple Kaposi's sarcoma papules, nodules, and stasis dermatitis. 



Figure 2 | (a) Lesion containing vascular structures of various sizes in the upper and lower dermis (hematoxylin and eosin stain [HE], $\times 40)(b)$ Mild nuclear hypertrophy and pleomorphism. Multiple vascular structures, some of which are fissured (HE, $\times 400)$.

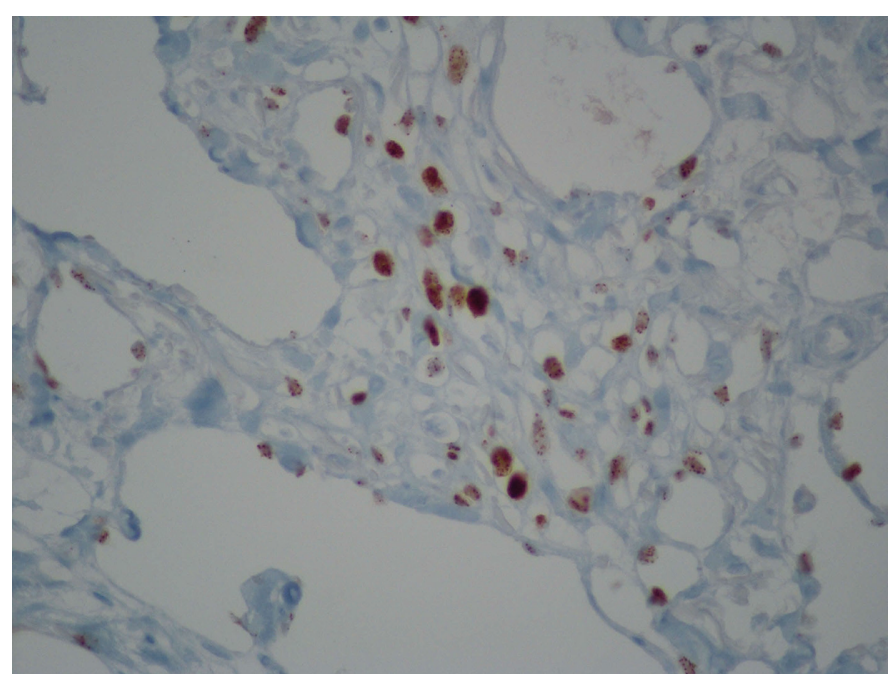

Figure 3 | Immunohistochemically confirmed HHV-8 (13B10, ×400).

\section{Discussion}

KS is a multifocal angioproliferative neoplasm characterized by reddish-purple-brown papules, plaques, and nodules on the lower extremities. It follows a chronic course and rarely affects organs other than the skin (1).

HHV-8 is the etiological factor responsible for the development of all types of KS. However, the condition develops in the presence of HHV-8 together with genetic, environmental, and immunological factors (6).

KS is considered a reactive disease. In the early stages, an increase in Th1-type inflammatory cytokines triggers the reactivation of HHV-8 infection, activation of endothelial cells, formation of fusiform cells, and angiogenesis induction. Disease progression requires the deficient immune control of viral escape mechanisms (6).

KS cases that developed with the use of oral steroids (2, 3), phototherapy (4), fumaric acid esters (1), infliximab, (7) and angiotensin-converting enzyme (ACE) inhibitors $(5,8)$ have been reported in patients with psoriasis. Tebbe et al. associated this concurrence with the presence of some human leukocyte antigen (HLA) loci (A1, DR5, DR7, and DR11) (3). In the case reported by Selvi et al., it was suggested that worsening of KS lesions despite discontinuation of systemic immunosuppressive therapy might have been related to intra-articular steroid therapy administered for psoriatic arthritis (2). Furthermore, in a KS patient with severe pso- riasis and a history of phototherapy, the authors suggested that the concurrent occurrence of psoriasis and KS may be related to a common genetic basis (HLA A1, DR5, DR7, and DR11), and that phototherapy may trigger KS formation by causing immunosuppression and virus activation (4).

Antihypertensive ACE inhibitors are known to have some immunomodulatory effects. In particular, ACE inhibitors inhibit interleukin (IL)-12 production. IL-12 is involved in cellular immunity and stimulates Th1-type immune response. Thus, the immunosuppressive effects of ACE inhibitors, immune changes caused by psoriasis, and the presence of HHV-8 infection may contribute to KS development (8). It should also be noted that, in addition to the triggering effect on psoriasis, ACE inhibitors can be a predisposing factor for KS development in psoriasis patients.

In psoriasis, Th1 cells and related cytokines play a major role in disease pathogenesis (1). Treatments that are intended to suppress the T cell response may trigger the development of iatrogenic KS by causing immunosuppression (5). In contrast to previously published cases, our patient did not use immunosuppressive drugs, was HIV-negative, and was diagnosed with classic KS. The chronic inflammatory condition in psoriasis can induce some neoplastic processes (9). In the present case, we considered that psoriasis, which is a chronic, inflammatory disease, might have triggered KS development by causing immune dysregulation.

Our case differs from other reports with concomitant venous insufficiency and stasis dermatitis. In angioproliferative lesions due to venous insufficiency and stasis dermatitis, acroangiodermatitis (pseudo-KS) is initially considered. In the present case, these two diseases were differentiated by means of histopathology and immunohistochemistry (HHV-8 positivity). Nevertheless, the preexisting psoriasis and stasis dermatitis in the patient concealed the KS lesions, and delayed the diagnosis and treatment.

Que et al. presented two cases of non-HIV-related Kaposi's sarcoma in the setting of chronic venous insufficiency and proposed that chronic venous insufficiency may be a predisposing factor for KS in immunocompetent patients (10). In the present case report we propose that KS development was associated with immune dysregulation due to psoriasis, but venous insufficiency may also play a role.

Some published cases have shown that KS lesions can mimic venous ulcers, arterial insufficiency, vascular ulcers, and chronic infected wounds (11). However, the concurrent occurrence of psoriasis, stasis dermatitis (venous ulcer), and KS has not been previously reported. 


\section{Conclusion}

In conclusion, we believe that careful monitoring is required for

\section{References}

1. Philipp S, Kokolakis G, Hund M, Witte E, Witte K, Kunz S, et al. Immunological changes in psoriasis patients under long-term treatment with fumaric acid esters: risk of Kaposi sarcoma occurrence? Eur J Dermatol. 2013;23:339-43.

2. Selvi E, De Stefano R, Manganelli S, Marcolongo R. Kaposi's sarcoma in psoriatic arthritis. Rheumatology (Oxford). 2003;42:389.

3. Tebbe B, Mayer-da-Silva A, Garbe C, von Keyserlingk HJ, Orfanos CE. Genetically determined coincidence of Kaposi sarcoma and psoriasis in an HIV-negative patient after prednisolone treatment: spontaneous regression 8 months after discontinuing therapy. Int J Dermatol. 1991;30:114-20.

4. Häring N, Strohal R, Kemmler N. Kaposi sarcoma in a psoriasis patient. J Dtsch Dermatol Ges. 2012;10:65-6.

5. Sorce M, Bongiorno MR. Simultaneous diagnosis of Kaposi's sarcoma, psoriasis, vitiligo and monoclonal gammopathy of undetermined significance in an immunocompetent patient. J Dermatol. 2013;40:479-80.

6. Fargnoli MC, Peris K, Frascione P, Barbati R, Anemona L, Uccini S, et al. Psoriasis, Kaposi's sarcoma and Hodgkin's disease in a patient with Down's syndrome. Dermatology. 2004;209:158-9. possible malignancies that might develop because of accompanying immune dysregulation in patients with psoriasis, even if they receive no immunosuppressive treatment or phototherapy.
7. Ursini F, Naty S, Mazzei V, Spagnolo F, Grembiale RD. Kaposi's sarcoma in a psoriatic arthritis patient treated with infliximab. Int Immunopharmacol. 2010;10:827-8.

8. Dervis E, Demirkesen C. Kaposi's sarcoma in a patient with psoriasis vulgaris. Acta Dermatovenerol Alp Pannonica Adriat. 2010;19:31-4.

9. Kimball AB, Schenfeld J, Accortt NA, Anthony MS, Rothman KJ, Pariser D. Incidence rates of malignancies and hospitalized infectious events in patients with psoriasis with or without treatment and a general population in the U.S.A.: 2005-09. Br J Dermatol. 2014;170:366-73.

10. Que SK, DeFelice T, Abdulla FR, Cassarino D, Patel RR. Non-HIV related Kaposi sarcoma in 2 Hispanic patients arising in the setting of chronic venous insufficiency. Cutis. 2015;95:E30-3.

11. Johnson EL, Pierpont YN, Donate G, Hiro MH, Mannari RJ, Strickland TJ, et al. Clinical challenge: cutaneous Kaposi's sarcoma of the lower extremity. Int Wound J. 2011;8:163-8. 